\title{
Colour constancy in natural images through colour naming and sensor sharpening
}

\author{
Javier Vazquez-Corral \\ Department de Tecnologies de la Informació i les Comunicacions, Universitat Pompeu Fabra, 08018, Barcelona, Catalonia, Spain \\ Advisor/s: Maria Vanrell, Graham Finlayson \\ Date and location of PhD thesis defense: 28 February 2011, Universitat Autònoma de Barcelona
}

Received 12th Feb 2014; accepted 25th May 2014

\section{Abstract}

Colour is derived from three physical properties: incident light, object reflectance and sensor sensitivities. Incident light varies under natural conditions; hence, recovering scene illuminant is an important issue in computational colour. One way to deal with this problem under calibrated conditions is by following three steps, 1) building a narrow-band sensor basis to accomplish the diagonal model, 2) building a feasible set of illuminants, and 3) defining criteria to select the best illuminant. In this work we focus on colour constancy for natural images by introducing perceptual criteria in the first and third stages.

To deal with the illuminant selection step, we hypothesize that basic colour categories can be used as anchor categories to recover the best illuminant. These colour names are related to how the human visual system has evolved to encode relevant natural colour statistics. Therefore the recovered image provides the best representation of the scene labelled with the basic colour terms. We demonstrate with several experiments how this selection criterion achieves current state-of-art results in computational colour constancy. In addition to this result, we psychophysically prove that usual angular error used in colour constancy does not correlate with human preferences, and we propose a new perceptual colour constancy evaluation.

The implementation of this selection criterion strongly relies on the use of a diagonal model for illuminant change. Then, the second contribution focuses on building an appropriate narrow-band sensor basis to represent natural images. We propose to use the spectral sharpening technique to compute a unique narrow-band basis optimized to represent a large set of natural reflectances under natural illuminants and given in the basis of human cones. The proposed sensors allow predicting unique hues and the World colour Survey data independently of the illuminant by using a compact singularity function. Additionally, we studied different families of sharp sensors to minimize different perceptual measures. This study brought us to extend the spherical sampling procedure from $3 \mathrm{D}$ to $6 \mathrm{D}$.

Several research lines remain still open, such as, measuring the effects of using the computed sharp sensors on the category hypothesis; or inserting spatial contextual information to improve category hypothesis. Finally,to explore how individual sensors can be adjusted to the colours in a scene.

Correspondence to: javier.vazquez@upf.edu

Recommended for acceptance by Alicia Fornés and Volkmar Frinken ELCVIA ISSN:1577-5097

Published by Computer Vision Center / Universitat Autònoma de Barcelona, Barcelona, Spain 